\title{
Theoretical and experimental studies of ultrasound-modulated optical tomography in biological tissue
}

\author{
Gang Yao and Lihong V. Wang
}

\begin{abstract}
Ultrasound-modulated optical tomography in biological tissue was studied both theoretically and experimentally. An ultrasonic beam was focused into biological tissue samples to modulate the laser light passing through the ultrasonic beam inside the tissue. The ultrasound-modulated laser light reflects the local optical and mechanical properties in the ultrasonic beam and permits tomographic imaging of biological tissues by scanning. Parallel detection of the speckle field formed by the transmitted laser light was implemented with the source-synchronous-illumination lock-in technique to improve the signalto-noise ratio. Two-dimensional images of biological tissues were successfully obtained experimentally with a laser beam at either normal or oblique incidence, which showed that ultrasound-modulated optical tomography depends on diffuse light rather than on ballistic light. Monte Carlo simulations showed that the modulation depth decreased much more slowly than the diffuse transmittance, which indicated the possibility that even thicker biological tissues can be imaged with this technique. (C) 2000 Optical Society of America
\end{abstract}

OCIS codes: $170.3880,120.6150,110.7050,110.7170$.

\section{Introduction}

Because it is noninvasive and nonionizing, optical imaging of biological tissues has been an active research area in recent years..$^{1,2}$ A number of optical imaging techniques, such as time-resolved optical imaging, ${ }^{3}$ frequency-domain optical imaging, ${ }^{4}$ and optical coherence tomography, ${ }^{5}$ have been proposed and studied. Contrast in optical imaging is generated by the optical absorption and scattering properties of biological tissues. Because biological tissues are highly scattering media, optical imaging techniques usually require sophisticated reconstruction algorithms if they are to achieve good imaging depth and reasonable resolution. Besides purely optical imaging techniques, hybrid methods that combine optical and ultrasonic techniques have been explored; they include the photoacoustic imaging, ${ }^{6-8}$ sonoluminescent tomography, ${ }^{9}$ and ultrasound-modulated optical

The authors are with the Optical Imaging Laboratory, Biomedical Engineering Program, Texas A \& M University, College Station, Texas 77843-3120. L. V. Wang's e-mail address is lwang@tamu.edu.

Received 21 July 1999; revised manuscript received 29 September 1999 .

0003-6935/00/040659-06\$15.00/0

(C) 2000 Optical Society of America tomography. ${ }^{10-13}$ Compared with light waves, ultrasonic waves scatter much less in biological tissues and can be used to furnish localization information directly for imaging. The hybrid techniques overcome the problem of poor localization of light in biological tissues caused by strong scattering.

In ultrasound-modulated optical tomography, some of the light is modulated by an ultrasonic wave inside the biological tissue and carries the ultrasonic frequency. Such tagged photons can be discriminated from background unmodulated photons, and their origins can be directly derived from the position of the ultrasonic column inside the tissue. Marks et al. ${ }^{10}$ reported the observation of ultrasound-modulated optical signals. Wang et al. ${ }^{11,12}$ developed ultrasoundmodulated optical tomography and obtained images in tissue-simulating phantoms. Wang and $\mathrm{Ku}^{14} \mathrm{em}-$ ployed a frequency-sweep technique to provide imaging resolution along the ultrasonic axis with a Fourier transform. A single-detector scheme was usually used in those studies. Recently Leveque et al. ${ }^{15} \mathrm{de}-$ veloped a scheme for parallel speckle detection, in which a CCD camera and a source-synchronized lock-in technique were used. They obtained onedimensional images of buried objects in real biological tissue (turkey breast). The CCD worked as a detector array in which each CCD pixel acted as a single speckle detector. Because there are usually tens of 
thousands pixels in a single CCD detector, the signalto-noise ratio (SNR) can be greatly improved by averaging of the signals from all the CCD pixels.

We further investigated imaging of biological tissues based on parallel detection of ultrasoundmodulated laser speckles by the synchronousillumination lock-in technique. For the first time to our knowledge, we acquired two-dimensional (2D) images of biological tissues with a laser beam at either normal or oblique incidence. We also studied ultrasound-modulated optical tomography by using Monte Carlo modeling. The number of photons passing through the ultrasonic column was calculated. These photons contribute to the modulated signal in ultrasound-modulated optical tomography. The changes in signal intensity with increasing sample thickness were studied. One-dimensional images of turbid media with buried objects were also simulated and provided some insight into ways to improve the image quality.

\section{Methods}

\section{A. Monte Carlo Simulation}

Although acousto-optics in clear media is a wellestablished subject, a quantitative understanding of the mechanisms of acousto-optic interactions in turbid media is still being sought. The modulation mechanisms include particle displacement and photon-phonon interactions. ${ }^{16,17}$ For both of the modulation mechanisms, only the photons that pass through the ultrasonic column can be modulated. The modulated signal intensity depends on two factors: the number of modulated photons and the modulation depth for each photon. The modulation depth, which is usually less than $10 \%$ in the experiments, is related to the modulation mechanisms and to the intensity of the ultrasonic wave. The number of modulated photons is related to the optical properties of the scattering medium and the size of the modulation volume. Because of the small size of the ultrasonic column, the number of modulated photons is usually quite small compared with the unmodulated background light. Detecting such a small signal requires improving the SNR. Several factors, such as the stability of the system and the coherence length of the light source, generate noise in the experimental measurements, which affects the stability and the contrast of the speckle pattern. Obviously, one can reduce such noise by improving the experimental conditions. The major intrinsic noise source in the experiments in the shot noise caused by the strong unmodulated background light. Therefore the number of modulated photons is an important variable that determines the SNR and the maximum thickness of biological tissues that can be imaged.

Monte Carlo modeling was used in our simulation studies; the detailed modulation mechanisms were not considered. We simply focused on the calculation of the number of photons that were passing through the ultrasonic column. In the simulation the tissue sample was modeled as a slab with a buried

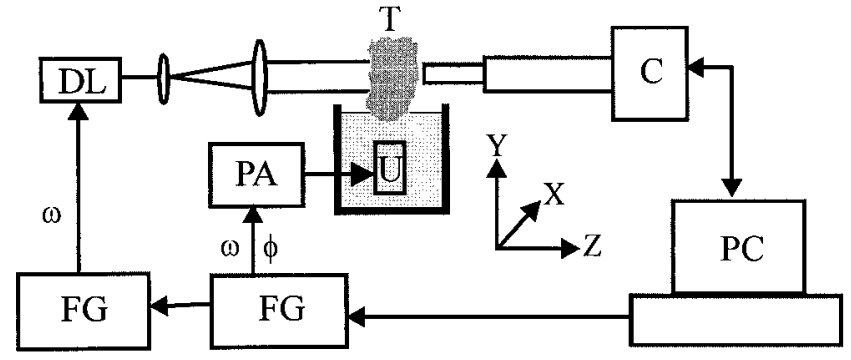

Fig. 1. Schematic sketch of the experimental setup: DL, diode laser; C, CCD camera; U, ultrasonic transducer; FG's, function generators; PA, power amplifier; $\mathrm{T}$, tissue sample; $\mathrm{PC}$, computer.

object. For simplicity, the object and the ultrasonic column were modeled as cylinders with heights of 0.6 and $2 \mathrm{~cm}$, respectively. The radius of the cylinder was assumed to be $1 \mathrm{~mm}$. Photons were launched and traced by the standard Monte Carlo method described elsewhere. ${ }^{18}$ A photon was labeled whenever it interacted with the ultrasonic column, either by being scattered in this column or by directly passing through it. The labeled transmitted photons were scored as the ac signal, and the unlabeled transmitted photons were scored as the dc signal. Only the photons transmitted within a circular disk of a 1-cm radius on the exit plane, which represented the detection area, were scored. The following values were used to simulate the optical properties of chicken-breast tissue ${ }^{19}$ : refractive index $n=1.33$, absorption coefficient $\mu_{a}=0.1 \mathrm{~cm}^{-1}$, scattering coefficient $\mu_{s}=20.0 \mathrm{~cm}^{-1}$, and anisotropy factor $g=0.9$.

\section{B. Experimental Study}

The tissue samples used in the experiments were skinless chicken-breast tissues acquired from a supermarket. The objects buried in the tissue were made from white rubber, which has good acoustic coupling with tissue and has little acoustic absorption.

The experimental setup for this study (Fig. 1) is similar to one reported previously. ${ }^{15}$ A coordinate system was set up for reference as shown in Fig. 1. The $Z$ axis lay along the optical axis pointing to the CCD camera. The $Y$ axis lay along the acoustic axis pointing to the sample. The $X$ axis was perpendicular to both the acoustic and the optical axes. A diode laser (Melles Griot, 56IMS667) was used as the light source. The laser delivered 14-mW 690-nm light when it was modulated at $1 \mathrm{MHz}$. The measured coherence length was $\sim 6 \mathrm{~cm}$. The ultrasound was generated by a focused ultrasonic transducer (Panametrics, V314) working at $1 \mathrm{MHz}$ with a $2.54-\mathrm{cm}$ focal length in water. The peak pressure at the focus was approximately $2 \times 10^{5} \mathrm{~Pa}$, well below the damage threshold for biological tissues. ${ }^{20}$ The laser beam was expanded to $2 \mathrm{~cm}$ in diameter and irradiated directly onto the tissue sample. The laser exposure was much less than the value set by the ANSI safety standard. ${ }^{21}$ The tissue sample was partially immersed in water for good acoustic coupling. 
The light transmitted from the tissue generated a speckle pattern, which was partially collected by a collection tube and detected by a high-speed 12-bit CCD camera (Dalsa, CA-D1-0256T). The exposure time of the CCD camera was adjusted to ensure that enough photons were detected. Two function generators (Stanford Research Systems, DS345) were used to excite the ultrasonic transducer and to modulate the diode laser. The two function generators shared the same time base for synchronization. The function generators and the CCD camera were controlled by a personal computer.

The signal in a single pixel can be represented as ${ }^{22}$

$$
I_{i} \propto I_{b}+I_{m} \cos \left(\phi+\phi_{i}\right),
$$

where $I_{b}$ is the background intensity, $I_{m}$ is the signal intensity related to the modulated ac signal, $\phi$ is the initial phase of the speckle, and $\phi_{i}$ is the phase delay of the signal applied to the ultrasonic transducer relative to the signal applied to the diode laser. The quantity $M=I_{m} / I_{b}$ is related to the modulation depth, which reflects the local optical and ultrasonic properties.

The source-synchronized lock-in detection scheme was implemented in the experiment. The diode laser and the ultrasonic transducer were modulated at the same frequency of $1 \mathrm{MHz}$. The phase delay $\phi_{i}$ was set sequentially to $0^{\circ}, 90^{\circ}, 180^{\circ}$, and $270^{\circ}$. The corresponding four frames of CCD images were acquired to calculate $M$ as follows:

$$
M=\frac{1}{2 I_{b}}\left[\left(I_{90^{\circ}}-I_{270^{\circ}}\right)^{2}+\left(I_{0^{\circ}}-I_{180^{\circ}}\right)^{2}\right]^{1 / 2} .
$$

The above calculation was performed for each pixel of the CCD camera. The data from all the $256 \times 256$ pixels of the CCD were then averaged to produce a single data point for the image. To further reduce the noise, we averaged 20 such measurements. To obtain a two-dimensional image, we scanned the tissue sample along the $X$ axis and scanned the ultrasonic transducer along the optical axis ( $Z$ axis). The relative lateral positions of the incident light with the ultrasonic column and the exit aperture were fixed in the scanning process.

\section{Results and Discussion}

\section{A. Simulation Results}

The speckle-detection scheme requires the coherence length of the laser to be greater than the optical path-length span of the transmitted photons. Figure 2 shows the simulated path-length distributions of the transmitted photons through a $3.0-\mathrm{cm}$-thick tissue sample. The path-length distributions for the ac and the dc photons have similar profiles. The path-length span at $50 \%$ of the peak is $\sim 7 \mathrm{~cm}$, which is comparable with the $6-\mathrm{cm}$ coherence length of the laser used in the experiments. It was also found that the ac photons typically pass through the ultrasonic column several times (1-6), which would enhance the ultrasonic modulation.

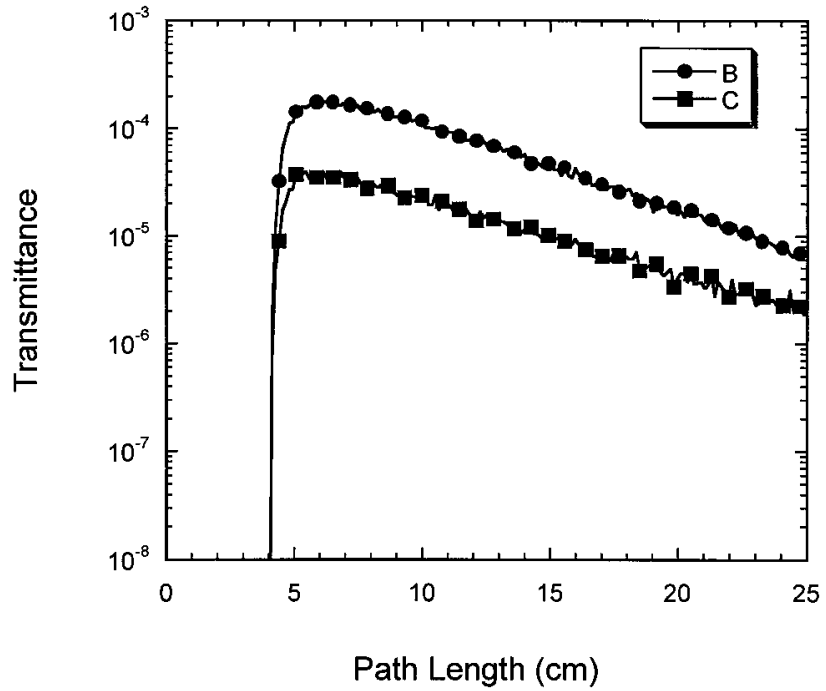

Fig. 2. Photon path-length distribution after transmission through a 3 -cm-thick tissue.

Figure 3 shows the simulated dc transmittance, ac transmittance, and modulation depth ac/dc at various sample thicknesses. There was no object buried in the medium. The ultrasonic column was assumed to be in the middle plane of the sample. In Fig. 3, the dc and ac transmittances decay almost exponentially. The dc transmittance is $\sim 10$ times less at the $3.0-\mathrm{cm}$ thickness than that at the $1.0-\mathrm{cm}$ thickness, whereas the ac transmittance is $\sim 20$ times less. However, the ac/dc ratio decreases by only a factor of 2. Because the modulation depth ac/dc is an important factor in determining the SNR, we suspect that the SNR decreases much more slowly than the diffuse transmittance as the tissue thickness increases.

One-dimensional scans along the $Z$ axis and the $X$ axis of $1.5-\mathrm{cm}$-thick chicken-breast tissue were simulated. An absorbing object was buried in the middle plane of the sample. Figure 4(a) shows the

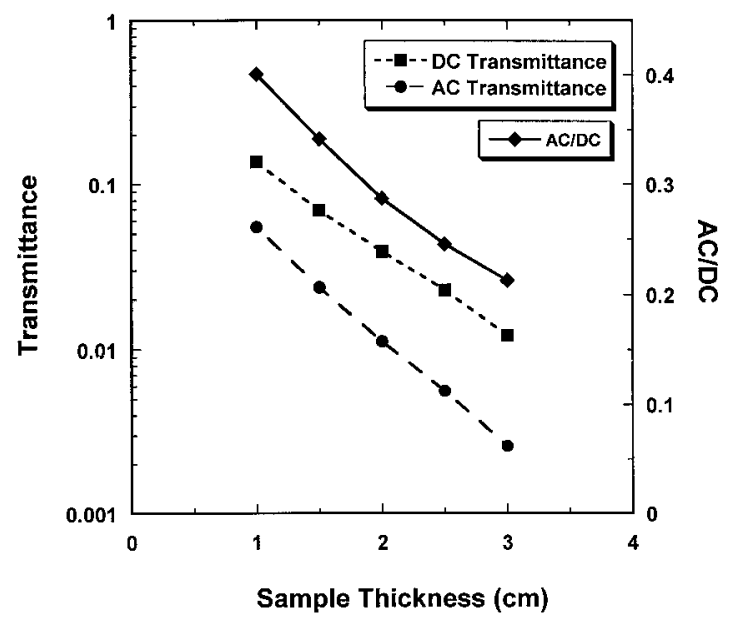

Fig. 3. ac and dc signal intensities versus tissue thickness. The ultrasonic column was in the middle plane of the sample slab. 

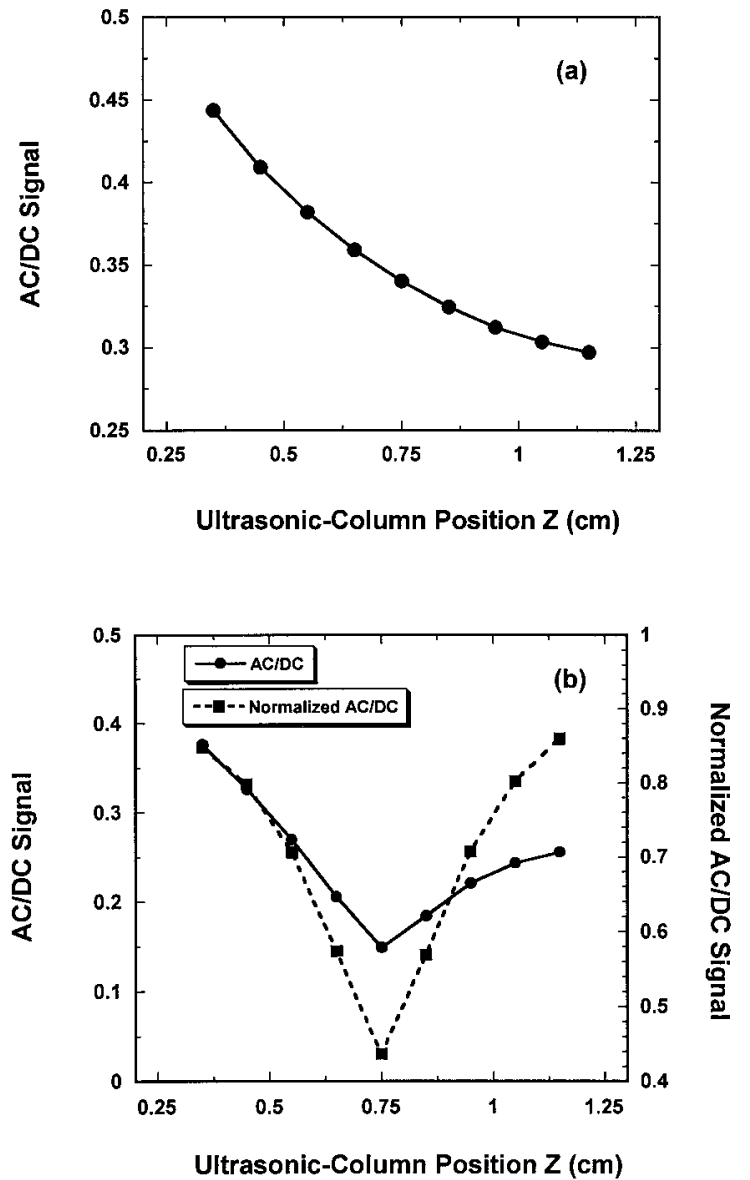

Fig. 4. Simulated $1 \mathrm{D}$ images along the $Z$ axis (optical axis). The light was incident at $Z=0$. (a) The ac/dc signal when the scan line was far away from the object, (b) the ac/dc signal when the scan line passed through the object.

result when the scan line was far way from the absorption object. The ac/dc signal decreases when the ultrasonic column moves away from the light source, which is caused by the internal photon distribution inside the tissue sample. The photon fluence is greater at positions near the incident light source, where they have more chances to pass through the ultrasonic column. Figure 4(b) shows the result when the scan line passed through the center of the object. Clearly, the signal is smaller when the ultrasonic column is coincident with the object. To compensate for the signal variation caused by the internal photon distribution, we can normalize the signal in Fig. 4(b) with the background signal in Fig. 4(a). The normalized signal is plotted as a dashed curve in Fig. 4(b). Obviously, the normalized result is much smoother and has higher contrast.

Figure 5 shows the result for the scan along the $X$ axis. The diffuse transmittance $(\mathrm{ac}+\mathrm{dc})$ is also plotted as a reference. As expected, the ac/dc curve has sharper resolution and better contrast than the diffuse-transmittance curve. Because the diameter of the ultrasonic column is $\sim 2 \mathrm{~mm}$, the object size measured from the ac/dc curve is greater than its actual size of $2 \mathrm{~mm}$. The spatial resolution in

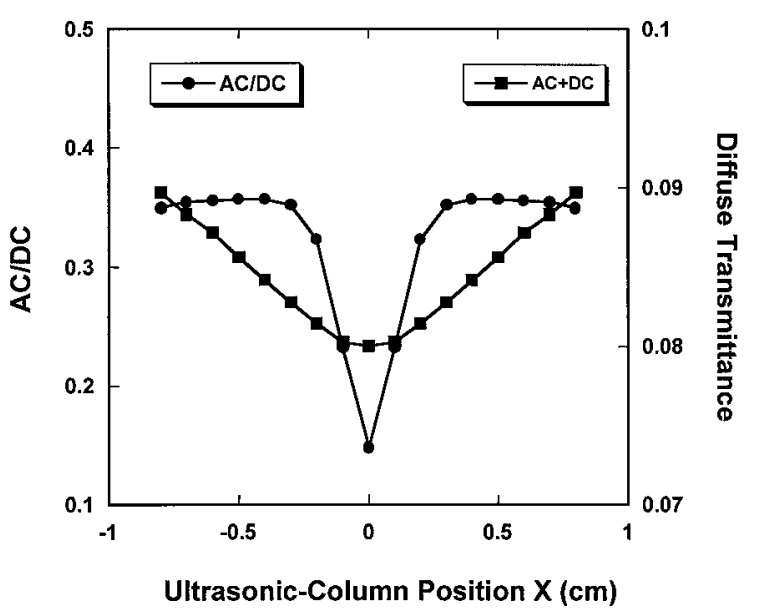

Fig. 5. Simulated $1 \mathrm{D}$ image along the $X$ axis with the scan line passing through the center of the object.

ultrasound-modulated optical imaging is determined by the size of the ultrasonic column.

\section{B. Experimental Results}

The speckle size was adjusted to match the pixel size of the CCD. The diameter of a single speckle $\Phi_{c}$ at the CCD could be calculated as $\Phi_{c}=\lambda z / \Phi_{e}$, where $\Phi_{e}$ was the effective diameter of the light exiting the sample surface, $\lambda$ was the laser wavelength, and $z$ was the distance from the exit sample surface to the CCD camera. In the experiments, the input diameter of the collection tube was $1.6 \mathrm{~cm}$ and the length from the input end to the CCD chip was $\sim 38 \mathrm{~cm}$. Therefore the speckle size was $\sim 16 \mu \mathrm{m}$, which was equal to the CCD pixel size.

To demonstrate the variations of the modulation depth relative to the detector area, we measured $M$ with various pixel-binning sizes of the CCD camera (Fig. 6). Each binned area (superpixel) acted as a single detector. The number of speckles in each single detector was proportional to $N_{b}^{2}$, where $N_{b} \times N_{b}$ was the binning size. Obviously, the modulation depth is greater for a smaller detector area. Theoretically, the overall modulation depth for the $N_{b}{ }^{2}$

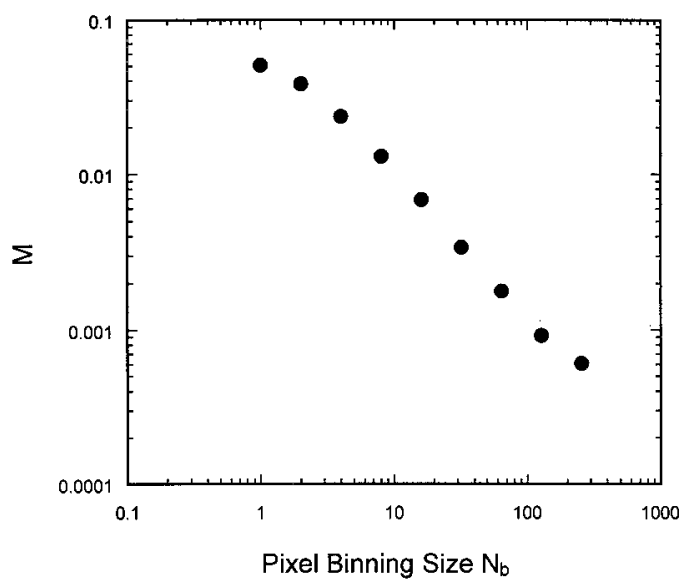

Fig. 6. Modulation depth $M$ versus CCD pixel-binning size. 

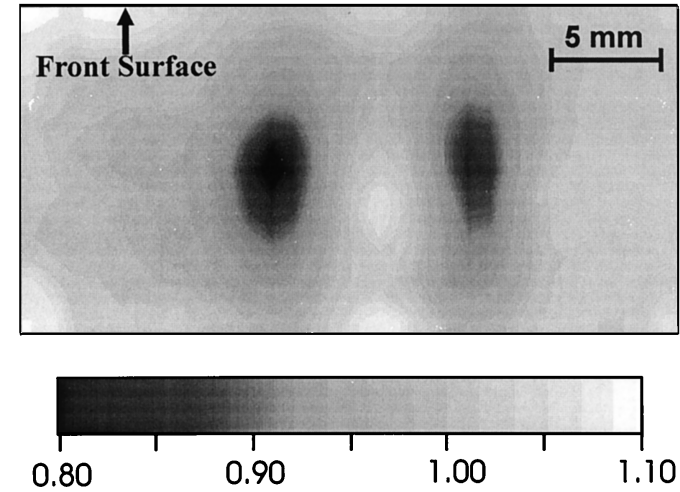

(a)

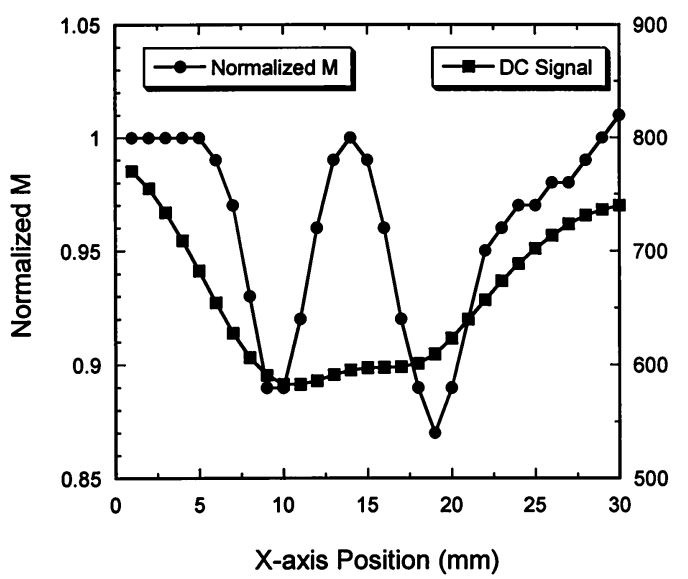

(b)

Fig. 7. Experimental images of 1.5-cm-thick chicken-breast tissue with a laser beam normally incident. (a) $2 \mathrm{D}$ cross-sectional image, (b) 1D image along the $X$ axis at the center of the sample. The front surface faced the laser beam.

speckles should be reduced by a factor of $N_{b}$ from the modulation depth for a single speckle.

Figure 7(a) shows a 2D ultrasound-modulated optical image obtained from 1.5 -cm thick chicken-breast tissue. Figure 7(b) shows a 1D plot corresponding to the scan line along the $X$ axis at the center of the tissue sample. The data in the image were normalized to the background value, and the $2 \mathrm{D}$ image was low-pass filtered. The sizes of the two objects were roughly $2.5 \mathrm{~mm} \times 2.8 \mathrm{~mm} \times 7.1 \mathrm{~mm}$ and $2.3 \mathrm{~mm} \times$ $2.7 \mathrm{~mm} \times 6.9 \mathrm{~mm}$, respectively. The two objects are clearly visible in the image. As for the simulated result (Fig. 5), the two objects are not discriminable in the dc-transmittance data, whereas they are sharply visible in the modulated data. The image resolution is approximately $2 \mathrm{~mm}$, which is determined by the focal size of the $1-\mathrm{MHz}$ ultrasonic transducer used in the experiments.

As previously measured, ${ }^{19}$ the reduced scattering coefficient of the chicken-breast tissue is $\sim 2 \mathrm{~cm}^{-1}$, and the absorption coefficient is $\sim 0.1 \mathrm{~cm}^{-1}$ at $\sim 700$ $\mathrm{nm}$. Therefore the penetration depth is $\sim 1 \mathrm{~cm}$, which indicates that ballistic photons passing through the $1.5-\mathrm{cm}$ tissue sample are not completely
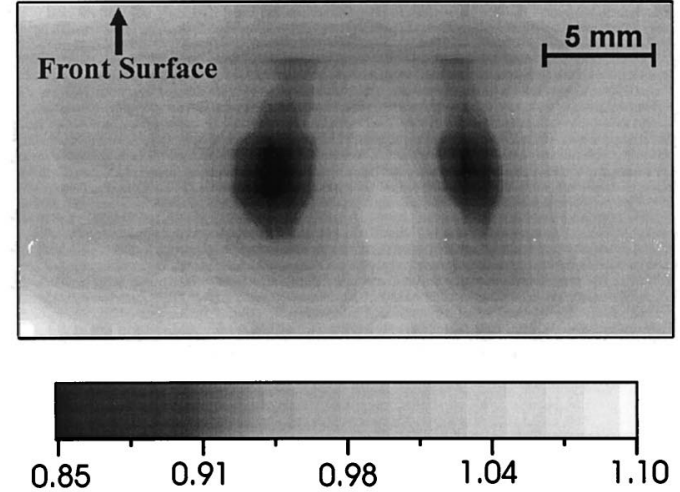

(a)

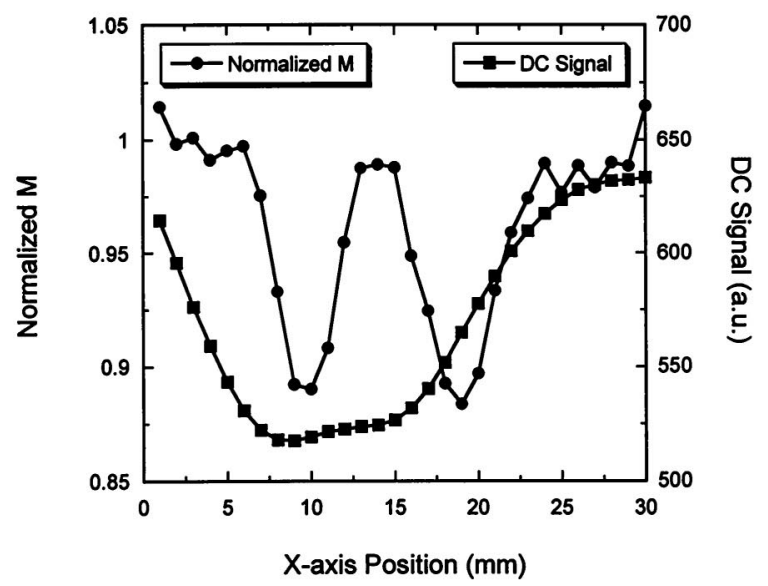

(b)

Fig. 8. Experimental images of 1.5-cm-thick chicken-breast tissue with a laser beam obliquely incident at $15^{\circ}$. (a) $2 \mathrm{D}$ crosssectional image, (b) 1D image along the $X$ axis at the center of the sample. The front surface faced the laser beam.

undetectable. To eliminate the effects of the purely ballistic photons, we acquired a 2D ultrasoundmodulated optical image with the light beam illuminating the sample obliquely at $15^{\circ}$ to the $Z$ axis (Fig. 8). The measured signal intensity was almost the same as that in the case of normal incidence. Compared with Fig. 7(a), Fig. 8 shows no observable degradation of image quality. From this result it is clear that the purely ballistic photons are not the major contributors of signal in our experiments and that ultrasound-modulated optical tomography depends on diffuse light.

Because of the small area of the CCD chip, only a small portion of the transmitted light was utilized in the image acquisition. To maintain a high enough SNR, the CCD camera needs to receive enough photons by using a long exposure time. The exposure time was set to $50 \mathrm{~ms}$, which was less than the $400-\mathrm{ms}$ correlation time of the speckle field as measured in our experiments. The image-acquisition time for a $2 \mathrm{D}$ image of $30 \times 15$ pixels was $\sim 30 \mathrm{~min}$. However, besides slowing down the data acquisition, a longer exposure time may cause the SNR to suffer 
from decorrelation of the speckle field if the exposure time becomes comparable with the correlation time of the speckle field. ${ }^{16}$ Apparently, one could overcome this problem by simply increasing the incident laser power.

\section{Conclusions}

Ultrasound-modulated optical tomography was studied both theoretically and experimentally. This technology has the potential to be used for early detection of breast cancers because it combines the contrast advantage of purely optical imaging and the resolution advantage of purely ultrasonic imaging, and optical properties are related to tissue constituents and their molecular conformations.

By implementing a parallel detection scheme with the synchronous-illumination lock-in technique, we obtained 2D images of 1.5-cm thick chicken-breast tissues with buried objects by using a low-power laser. The number of photons that passed through the ultrasonic column was calculated by Monte Carlo simulations. These photons are the source of the modulated signal. The Monte Carlo simulation results show that, although the total ac transmittance decreases $\sim 9$ times when the tissue thickness is increased from 1.5 to $3.0 \mathrm{~cm}$, the ac/dc ratio decreases only $\sim 1.6$ times. Therefore, by increasing the incident laser power by a factor of 10 from the current power, we expect to be able to image chicken-breast tissue with a thickness of greater than $3 \mathrm{~cm}$. Furthermore, if a CCD camera with more pixels and higher sensitivity is used, it is possible to increase the imaging acquisition speed further and avoid the speckle-decorrelation problem.

Spatial resolution in ultrasound-modulated optical tomography is determined by the size of the ultrasonic column. A spatial resolution of $2 \mathrm{~mm}$ was obtained in our experiments with a $1-\mathrm{MHz}$ focused ultrasonic transducer. Higher resolution could be achieved by use of an ultrasonic transducer with high frequency and a greater numerical aperture.

In our current experimental setup, each data point comes from an integrated contribution of signals along the ultrasonic column. The spatial resolution along the ultrasonic axis will not be good because the focal depth of the ultrasonic transducer is $\sim 2 \mathrm{~cm}$ in the current setup. However, it is possible to achieve spatial resolution along the ultrasonic axis and to obtain three-dimensional images in turbid media by use of image-reconstruction techniques.

This project was sponsored in part by National Institutes of Health grants R29 CA68562, R01 CA71980, and R21 CA83760 and by National Science Foundation grant BES-9734491.

\section{References}

1. R. R. Alfano and J. G. Fujimoto, eds., Advances in Optical Imaging and Photon Migration, Vol. 2 of OSA Trends in Optics and Photonics Series (Optical Society of America, Washington, D.C., 1996).
2. B. Chance and R. R. Alfano, eds., Optical Tomography and Spectroscopy of Tissue: Theory, Instrumentation, Model, and Human Studies II, Proc. SPIE 2979 (1997).

3. J. C. Hebden and D. T. Delpy, "Enhanced time-resolved imaging with a diffusion model of photon transport," Opt. Lett. 9, 311-313 (1994).

4. M. A. O'Leary, D. A. Boas, B. Chance, and A. G. Yodh, "Experimental images of heterogeneous turbid media by frequency-domain diffusing-photon tomography," Opt. Lett. 20, 426-428 (1995).

5. A. F. Fercher, "Optical coherence tomography," J. Biomed. Opt. 1, 157-173 (1996).

6. R. A. Kruger and P. Liu, "Photoacoustic ultrasound: theory," in Laser-Tissue Interaction V, S. L. Jacques, ed., Proc. SPIE 2134A, 114-118 (1994).

7. A. A. Oraevsky, R. O. Esenaliev, S. L. Jacques, and F. K. Tittel, "Laser optic-acoustic tomography for medical diagnostics: principles," in Biomedical Sensing, Imaging, and Tracking Technologies I, R. A. Lieberman, H. Podbielska, and T. Vo-Dinh, eds., Proc. SPIE 2676, 22-31 (1996).

8. C. G. A. Hoelen, F. F. M. de Mul, R. Pongers, and A. Dekker, "Three-dimensional photoacoustic imaging of blood vessels in tissue," Opt. Lett. 23, 648-650 (1998).

9. L.-H. Wang and Q. Shen, "Sonoluminescence tomography of turbid media," Opt. Lett. 23, 561-563 (1998).

10. F. A. Marks, H. W. Tomlinson, and G. W. Brooksby, "Comprehensive approach to breast cancer detection using light: photon localization by ultrasound modulation and tissue characterization by spectral discrimination," in Photon Migration and Imaging in Random Media and Tissue, B. Chance and R. R. Alfano, eds., Proc. SPIE 1888, 500-510 (1993).

11. L.-H. Wang, S. L. Jacques, and X. Zhao, "Continuous-wave ultrasonic modulation of scattered laser light to image objects in turbid media," Opt. Lett. 20, 629-631 (1995).

12. L.-H. Wang and X. Zhao, "Ultrasound-modulated optical tomography of absorbing objects buried in dense tissuesimulating turbid media," Appl. Opt. 36, 7277-7282 (1997).

13. M. Kempe, M. Larionov, D. Zaslavsky, and A. Z. Genack, "Acousto-optic tomography with multiple scattered light," J. Opt. Soc. Am. 14, 1151-1158 (1997).

14. L.-H. Wang, and G. Ku, "Frequency-swept ultrasoundmodulated optical tomography of scattering media," Opt. Lett. 23, 975-977 (1998).

15. S. Leveque, A. C. Boccara, M. Lebec, and H. Saint-Jalmes, "Ultrasonic tagging of photon paths in scattering media: parallel speckle modulation processing," Opt. Lett. 24, 181-183 (1999).

16. W. Leutz and G. Maret, "Ultrasonic modulation of multiply scattered light," Phys. B 204, 14-19 (1995).

17. L.-H. Wang, "Ultrasonic modulation of scattered light in turbid media and a potential novel tomography in biomedicine," Photochem. Photobiol. 67, 41-49 (1998).

18. L.-H. Wang, S. L. Jacques, and L.-Q. Zheng, "MCML-Monte Carlo modeling of photon transport in multi-layered tissue," Comput. Methods Programs Biomed. 47, 131-146 (1995).

19. G. Marquez, L.-H. Wang, S.-P. Lin, J. A. Schwartz, and S. L. Thomsen, "Anisotropy in the absorption and scattering spectra of chicken breast tissue," Appl. Opt. 37, 798-805 (1998).

20. T. A. Whittingham, "The safety of ultrasound," Imaging 6 , 33-51 (1994).

21. American National Standards Institute, American National Standard for the Safe Use of Lasers, Standard Z136.1-1993 (ANSI, Inc., New York, 1993).

22. P. Gleyzes, F. Guernet, and A. C. Boccara, "Picometric profilometry. II. Multidetector approach and multiplexed lock-in detection,” J. Opt. 26, 251-265 (1995). 